\title{
Semiclassical evolution of the spectral curve in the normal random matrix ensemble as Whitham hierarchy
}

\author{
R. Teodorescu ${ }^{\mathrm{a}, 1}$, E. Bettelheim ${ }^{\mathrm{b}, 2}$, O. Agam $^{\mathrm{b}}$, A. Zabrodin ${ }^{\mathrm{c}}$, \\ and P. Wiegmann ${ }^{\mathrm{d}}$ \\ ${ }^{a}$ James Frank Institute, University of Chicago, 5640 S. Ellis Ave. Chicago, IL \\ 60637, USA. \\ ${ }^{\mathrm{b}}$ Racah Institute of Physics, Hebrew University, Givat Ram, Jerusalem, Israel \\ 91904 \\ ${ }^{\mathrm{c}}$ Institute of Biochemical Physics, Kosygina str. 4, 117334 Moscow, Russia, also at \\ ITEP, Bol. Cheremushkinskaya str. 25, 117259 Moscow, Russia \\ d James Frank Institute, Enrico Fermi Institute, University of Chicago, $5640 \mathrm{~S}$. \\ Ellis Ave. Chicago, IL 60615, USA, and the Landau Institute, Moscow, Russia
}

\begin{abstract}
We continue the analysis of the spectral curve of the normal random matrix ensemble, introduced in an earlier paper. Evolution of the full quantum curve is given in terms of compatibility equations of independent flows. The semiclassical limit of these flows is expressed through canonical differential forms of the spectral curve. We also prove that the semiclassical limit of the evolution equations is equivalent to Whitham hierarchy.
\end{abstract}

Key words: Integrable Systems, Random Matrix Theory PACS: 02.50.Ey, 02.30.-f

Email addresses: rteodore@uchicago.edu (R. Teodorescu), eldadb@phys.huji.ac.il (E. Bettelheim), agam@phys.huji.ac.il (O. Agam), zabrodin@itep.ru (A. Zabrodin), wiegmann@uchicago.edu (P. Wiegmann).

1 Present address: Columbia University, Department of Physics, 538 W. 120th St., New York, NY 10027, USA.

2 Present address: James Frank Institute, University of Chicago, 5640 S. Ellis Ave. Chicago, IL 60637, USA.

Preprint submitted to Nuclear Physics B 27 October 2018 


\section{Introduction}

Continuing the study of the normal random matrix ensemble, presented in the series of papers $[1,2,3,4]$, we explore the evolution of the spectral curve with respect to all the independent flows.

In the first part of the paper, we review the basic concepts of the theory, relevant to many different developments, from integrability of non-linear differential equations to supersymmetric Yang-Mills theory $[5,6,7,8,9,10,11]$.

In the second part, we derive the evolution equations and prove that their semiclassical limit is the universal Whitham hierarchy associated with the complex curve. We also notice that the Whitham hierarchy is identical to the set of equations which describe Laplacian growth processes - unstable dynamics of an interface between two immiscible phases.

\subsection{Normal matrix ensemble}

The following is a short review of normal random matrix theory. Normal matrices $M$ commute with their hermitian conjugate: $\left[M, M^{\dagger}\right]=0$, so that both $M$ and $M^{\dagger}$ can be diagonalized simultaneously, and have complex eigenvalues. The statistical weight of the normal matrix ensemble is given through a general potential $W\left(M, M^{\dagger}\right)[12]$ :

$$
e^{\frac{1}{\hbar} \operatorname{tr} W\left(M, M^{\dagger}\right)} d \mu(M) .
$$

Here $\hbar$ is a parameter, and the measure of integration over normal matrices is induced by the flat metric on the space of all complex matrices. Using the standard procedure [13], angular degrees of freedom are integrated out, leading to the joint probability distribution of eigenvalues $z_{1}, \ldots, z_{N}$, where $N$ is size of the matrix:

$$
\frac{1}{N ! \tau_{N}}\left|\Delta_{N}(z)\right|^{2} \prod_{j=1}^{N} e^{\frac{1}{\hbar} W\left(z_{j}, \bar{z}_{j}\right)} d^{2} z_{j}
$$

Here $\Delta_{N}(z)=\operatorname{det}\left(z_{j}^{i-1}\right)_{1 \leq i, j \leq N}=\prod_{i>j}^{N}\left(z_{i}-z_{j}\right)$ is the Vandermonde determinant, and

$$
\tau_{N}=\frac{1}{N !} \int\left|\Delta_{N}(z)\right|^{2} \prod_{j=1}^{N} e^{\frac{1}{\hbar} W\left(z_{j}, \bar{z}_{j}\right)} d^{2} z_{j}
$$

is a normalization factor, the partition function of the matrix model.

We consider the case where the potential $W$ has the form

$$
W=-|z|^{2}+V(z)+\overline{V(z)}
$$


where $V(z)$ is a holomorphic function in a domain which includes the support of eigenvalues. We also assume that the field

$$
A(z)=\partial_{z} V(z)
$$

(a "vector potential") is a globally defined meromorphic function.

In a proper large $N$ limit ( $\hbar \rightarrow 0, N \hbar$ fixed), the eigenvalues of matrices occupy a connected domain $D$ in the complex plane, or, in general, several disconnected domains. We refer to the connected components $D_{\alpha}$ of the domain $D$ as droplets.

In the case of algebraic domains [4], the eigenvalues are distributed with the density $\rho=-\frac{1}{4 \pi} \Delta W$, where $\Delta=4 \partial_{z} \partial_{\bar{z}}$ is the 2-D Laplace operator [14]. For the potential (4), the density is uniform.

Boundary components of the droplets form a real section of a complex curve, defined in the following way: let us represent the boundary of the domain as a real curve $F(x, y)=0$. If the vector potential $A(z)$ is a meromorphic function (we always assume that this is the case), the function $F$ can be chosen to be an irreducible polynomial. Then we rewrite it in holomorphic coordinates as

$$
F\left(\frac{z+\bar{z}}{2}, \frac{z-\bar{z}}{2 i}\right)=f(z, \bar{z})
$$

and treat $z$ and $\bar{z}$ as independent complex coordinates $z, \tilde{z}$. The equation $f(z, \tilde{z})=0$ defines a complex curve. This curve is a finite-sheet covering of the $z$-plane. The single-valued function $\tilde{z}(z)$ on the curve is a multivalued function on the $z$-plane. Making cuts, one can fix single-valued branches of this function. The boundary of the domain is a section of the curve by the plane where $\tilde{z}$ is complex conjugate of $z$.

Similarly to the hermitian matrix ensemble $[5,10,11]$, the complex curve of the normal matrix ensemble is characterized by the potential $W$ (or by the "vector potential" $A(z)$ ), and by a set of $g+1$ integers $\nu_{\alpha}$ (not necessarily positive), where $g$ is genus of the curve. The integers are subject to the constraint $\sum_{\alpha=0}^{g} \nu_{\alpha}=N$. If they are all positive, then they are proportional to the areas of the droplets of uniformly distributed eigenvalues. In this case, every droplet contains $\nu_{\alpha}$ eigenvalues.

As one varies the potential and the filling factors $\nu_{\alpha}$, the curve and the interface bounding the droplets evolve. Parameters of the potential (for example, poles and residues of the meromorphic function (5)) and filling factors are deformation parameters and parameters of growth. They are coordinates in the moduli space of the complex curves. An infinitesimal variation of the potential generates correlation functions of the ensemble [14]. 


\section{Spectral curve and wave functions}

In this section we specify the potential to be of the form (4), and introduce the set of biorthogonal wave functions

$$
\psi_{n}(z)=e^{-\frac{|z|^{2}}{2 \hbar}+\frac{1}{\hbar} V(z)} P_{n}(z), \quad \text { and } \quad \chi_{n}(z)=e^{\frac{1}{\hbar} V(z)} P_{n}(z)
$$

where the holomorphic functions $\chi_{n}(z)$ are orthonormal in the complex plane with the weight $e^{-|z|^{2} / \hbar}$. Like traditional orthogonal polynomials, the biorthogonal polynomials $P_{n}$ (and the corresponding wave functions) obey a set of differential equations with respect to the argument $z$, and recurrence relations with respect to the degree $n$. Similar equations for two-matrix models are discussed in numerous papers (see, e.g., [15]).

In the basis (7), multiplication by $z$ is represented by the $L$-operator (the Lax operator):

$$
L_{n m} \chi_{m}(z)=z \chi_{n}(z)
$$

(summation over repeated indices is implied). Obviously, $L$ is a lower triangular matrix with one adjacent upper diagonal, $L_{n m}=0$ as $m>n+1$. Similarly, the differentiation $\partial_{z}$ is represented by an upper triangular matrix with one adjacent lower diagonal. Integrating by parts the matrix elements of the $\partial_{z}$, one finds:

$$
\left(L^{\dagger}\right)_{n m} \chi_{m}=\hbar \partial_{z} \chi_{n},
$$

where $L^{\dagger}$ is the hermitian conjugate operator.

The matrix elements of $L^{\dagger}$ are

$$
\left(L^{\dagger}\right)_{n m}=\bar{L}_{m n}=A\left(L_{n m}\right)+\int e^{\frac{1}{\hbar} W} \bar{P}_{m}(\bar{z}) \partial_{z} P_{n}(z) d^{2} z
$$

where the last term is a lower triangular matrix. The latter can be written through negative powers of the Lax operator. Writing $\partial_{z} \log P_{n}(z)=\frac{n}{z}+$ $\sum_{k>1} v_{k}(n) z^{-k}$, one represents $L^{\dagger}$ in the form

$$
L^{\dagger}=A(L)+(\hbar n) L^{-1}+\sum_{k>1} v^{(k)} L^{-k}
$$

where $v^{(k)}$ and $(\hbar n)$ are diagonal matrices with elements $v_{n}^{(k)}$ and $(\hbar n)$. The

coefficients $v_{n}^{(k)}$ are determined by the condition that lower triangular matrix elements of $A\left(L_{n m}\right)$ are cancelled.

In order to emphasize the structure of the operator $L$, we write it in the basis of the shift operator $\hat{w}$ such that $\hat{w} f_{n}=f_{n+1} \hat{w}$ for any sequence $f_{n}$. Acting on the wave function, we have:

$$
\hat{w} \chi_{n}=\chi_{n+1} \text {. }
$$


In the $n$-representation, the operators $L, L^{\dagger}$ acquire the form

$$
L=r_{n} \hat{w}+\sum_{k \geq 0} u_{n}^{(k)} \hat{w}^{-k}, \quad L^{\dagger}=\hat{w}^{-1} r_{n}+\sum_{k \geq 0} \hat{w}^{k} \bar{u}_{n}^{(k)} .
$$

Acting on $\chi_{n}$, we have the commutation relation ("the string equation")

$$
\left[L, L^{\dagger}\right]=\hbar .
$$

This is the compatibility condition of Eqs. (8) and (9).

Equations (11) and (12) completely determine the coefficients $v_{n}^{(k)}, r_{n}$ and $u_{n}^{(k)}$. The first one connects the coefficients to the parameters of the potential. The second equation is used to determine how the coefficients $v_{n}^{(k)}, r_{n}$ and $u_{n}^{(k)}$ evolve with $n$. In particular, its diagonal part reads

$$
n \hbar=r_{n}^{2}-\sum_{k \geq 1} \sum_{p=1}^{k}\left|u_{n+p}^{(k)}\right|^{2} .
$$

\subsection{Finite dimensional reductions}

If the vector potential $A(z)$ is a rational function, the coefficients $u_{n}^{(k)}$ are not all independent. The number of independent coefficients equals the number of independent parameters of the potential. For example, if the holomorphic part of the potential, $V(z)$, is a polynomial of degree $d$, the series (11) are truncated at $k=d-1$.

In this case, the semi-infinite system of linear equations (9) and the recurrence relations (8) can be cast in the form of a set of finite dimensional differential equations, whose coefficients are rational functions of $z$, one system for every $n>0$. The system of differential equations generalizes the CristoffelDaurboux second order differential equation valid for orthogonal polynomials. This fact has been observed in recent papers $[16,17]$ for biorthogonal polynomials emerging in the hermitian two-matrix model with a polynomial potential. It is applicable to our case (holomorphic biorthogonal polynomials) as well.

The semi-infinite set $\left\{\chi_{0}, \chi_{1}, \ldots\right\}$ is then decomposed as a "bundle" of $d$ dimensional vectors

$$
\underline{\chi}(n)=\left(\chi_{n}, \chi_{n+1}, \ldots, \chi_{n+d-1}\right)^{\mathrm{t}}
$$

(the index $\mathrm{t}$ means transposition, so $\chi$ is a column vector). The dimension of the vector is the number of poles of $\overline{A(z)}$ plus one. Each vector obeys a closed $d$-dimensional linear differential equation

$$
\hbar \partial_{z} \underline{\chi}(n)=\mathcal{L}_{n}(z) \underline{\chi}(n),
$$


where the $d \times d$ matrix $\mathcal{L}_{n}$ is a "projection" of the operator $L^{\dagger}$ onto the $n$-th $d$-dimensional space. Matrix elements of the $\mathcal{L}_{n}$ are rational functions of $z$ having the same poles as $A(z)$ and also a pole at the point $\overline{A(\infty)}$. If $A(z)$ is a polynomial, all these poles accumulate to a multiple pole at infinity.

\subsection{Spectral curve}

The semiclassical asymptotics of solutions to Eq. (14), as $\hbar \rightarrow 0$, are found by solving the eigenvalue problem for the matrix $\mathcal{L}_{n}(z)$ [18]. More precisely, the basic object of the the asymptotic analysis is the spectral curve [19] of the matrix $\mathcal{L}_{n}$, which is defined, for every integer $n>0$, by the secular equation $\operatorname{det}\left(\mathcal{L}_{n}(z)-\tilde{z}\right)=0$ (here $\tilde{z}$ means $\tilde{z} \cdot \mathbf{1}$, where $\mathbf{1}$ is the unit $d \times d$ matrix). It is clear that the left hand side of the secular equation is a polynomial in $\tilde{z}$ of degree $d$. We define the spectral curve by an equivalent equation

$$
f_{n}(z, \tilde{z})=a(z) \operatorname{det}\left(\mathcal{L}_{n}(z)-\tilde{z}\right)=0
$$

where the factor $a(z)$ is added to make $f_{n}(z, \tilde{z})$ a polynomial in $z$ as well. The factor $a(z)$ then has zeros at the points where poles of the matrix function $\mathcal{L}(z)$ are located. It does not depend on $n$.

The spectral curve (15) is characterized by the antiholomorphic involution. In the coordinates $z, \tilde{z}$, the involution reads $(z, \tilde{z}) \mapsto(\overline{\tilde{z}}, \bar{z})$. This simply means that the secular equation $\operatorname{det}\left(\overline{\mathcal{L}}_{n}(\tilde{z})-z\right)=0$ for the matrix $\overline{\mathcal{L}}_{n}(\tilde{z}) \equiv \overline{\mathcal{L}_{n}(\overline{\tilde{z}})}$ defines the same curve. Therefore, the polynomial $f_{n}$ takes real values for $\tilde{z}=\bar{z}$ :

$$
f_{n}(z, \bar{z})=\overline{f_{n}(z, \bar{z})}
$$

Points of the real section of the curve $(\tilde{z}=\bar{z})$ are fixed points of the involution.

\subsection{Schwarz function}

The polynomial $f_{n}(z, \bar{z})$ can be factorized in two ways:

$$
f_{n}(z, \bar{z})=a(z)\left(\bar{z}-S_{n}^{(1)}(z)\right) \ldots\left(\bar{z}-S_{n}^{(d)}(z)\right),
$$

where $S_{n}^{(i)}(z)$ are eigenvalues of the matrix $\mathcal{L}_{n}(z)$, or

$$
f_{n}(z, \bar{z})=\overline{a(z)}\left(z-\bar{S}_{n}^{(1)}(\bar{z})\right) \ldots\left(z-\bar{S}_{n}^{(d)}(\bar{z})\right),
$$

where $\bar{S}_{n}^{(i)}(\bar{z})$ are eigenvalues of the matrix $\overline{\mathcal{L}}_{n}(\bar{z})$. One may understand them as different branches of a multivalued function $S(z)$ (respectively, $\bar{S}(z)$ ) on 
the plane (here we do not indicate the dependence on $n$, for simplicity of the notation). It then follows that $S(z)$ and $\bar{S}(z)$ are mutually inverse functions:

$$
\bar{S}(S(z))=z
$$

An algebraic function with this property is called the Schwarz function. The equation $f(z, S(z))=0$ defines a complex curve with an antiholomorphic involution. An upper bound for genus of this curve is $g=(d-1)^{2}$, where $d$ is the number of branches of the Schwarz function. The real section of this curve is the set of all fixed points of the involution. It consists of a number of contours on the plane (and possibly a number of isolated points, if the curve is not smooth). The structure of this set is known to be complicated. Depending on the coefficients of the polynomial, the number of disconnected contours in the real section may vary from 0 to $g+1$. If the contours divide the complex curve into two disconnected "halves", or sides (related by the involution), then the curve can be realized as the Schottky double $[20,21,22]$ of one of these sides. Each side is a Riemann surface with a boundary.

The Schwarz function on the physical sheet is a particular root, say $S_{n}^{(1)}(z)$, of the polynomial $f_{n}(z, \tilde{z})$ (see $(17)$ ). It follows from (10) that this root is selected by the requirement that it has the same poles and residues as the potential $A$.

The formal ${ }^{3}$ semiclassical asymptote of equation (14), in the leading order in $\hbar$, is

$$
\chi_{n} \sim e^{\frac{1}{\hbar} \int^{z} d \Omega_{n}^{(1)}} .
$$

Here

$$
d \Omega_{n}^{(1)}=S_{n}^{(1)} d z .
$$

The differential $d \Omega^{(1)}$ is a physical branch of the generating differential on the curve (see below).

The semiclassical asymptotics was discussed in more details in [4]. To next order in $\hbar$, it reads

$$
\psi(z) \sqrt{d z} \sim \sqrt{W^{(\infty, \bar{\infty})}(z)} e^{-\frac{1}{\hbar}\left(\frac{|z|^{2}}{2}-\int_{\xi_{0}}^{z} S(z) d z\right)} .
$$

\subsection{The generating differential}

The meromorphic differential

$$
d \Omega=S(z) d z
$$

$\overline{3}$ This formal expression ignores the Stokes phenomenon. 
plays an important role. It is called generating differential [3,23]. On the physical sheet it has the same poles and residues as the differential $A d z$ :

$$
d \Omega=A d z+S_{-}(z) d z
$$

In the following, we use the following properties of the generating differential.

(i) The periods over a-cycles (boundaries of the droplets) are purely imaginary, and are integer multiples of $2 \pi i$. They compute areas of the droplets:

$$
\nu_{\alpha}=\frac{1}{2 \pi i \hbar} \oint_{\mathbf{a}_{\alpha}} d \Omega
$$

The filling factors of physical droplets (belonging to the physical sheet) are positive.

(ii) The real part of the integral of the differential $(\bar{z}-S(z)) d z$ from some fixed point $\xi_{0}$ to a point on the boundary of a droplet (a point on a a-cycle) has the same value for all points of the boundary:

$$
\phi_{\alpha}=-|z|^{2}+2 \mathcal{R} e \int_{\xi_{0}}^{z} d \Omega=\text { const }, \quad \text { for all } z \in \mathbf{a}_{\alpha} .
$$

This quantity does not depend on $z$, but does depend on $\xi_{0}$ unless $\xi_{0}$ is on the boundary. However, the difference $\phi_{\alpha}-\phi_{\beta}$ depends on the a-cycles only. It is equal to a b-period of the differential $d \Omega$ :

$$
\phi_{\alpha}-\phi_{\beta}=\oint_{\mathbf{b}_{\alpha \beta}} d \Omega
$$

where $\mathbf{b}_{\alpha \beta}$ is a cycle connecting $\mathbf{a}_{\alpha}$ and $\mathbf{a}_{\beta}$ cycles.

For proofs and more details, see Ref. [3].

Periods over b-cycles $\phi_{\alpha}-\phi_{0}$ play a role of chemical potentials for the filling factors. Here 0 denotes a chosen reference droplet. One can use chemical potentials to characterize evolution of the curve instead of filling factors.

\section{Evolution of the spectral curve}

\subsection{Evolution of the quantum curve}

Evolution of wave functions of the matrix ensembles with respect to a change of the potential is the subject of vast literature. Most of it deals with orthogonal polynomials of the hermitian ensemble (see, e.g., [24]). Our case is similar to 
the case of biorthogonal polynomials appearing in two-matrix ensembles [15]. We represent the evolution equations through variational derivatives $\delta / \delta A(z)$ with respect to the holomorphic part of the potential. This does not imply any particular parametrization of the potential. The standard result reads (cf. [23]):

$$
\hbar \frac{\delta}{\delta A(\zeta)} \psi_{n}(z)=H_{n m}(\zeta) \psi_{m}(z)
$$

where

$$
H(\zeta)=-[\log (\zeta-L)]_{+}-\frac{1}{2}[\log (\zeta-L)]_{0}-\frac{1}{2} \log \zeta
$$

and the notation $[\ldots]_{+, 0}$ means the upper triangular or diagonal part of the matrix. This formula reflects the triangular structure of the $L$-operator encoded by Eq.(10)

$$
L^{\dagger}=A(L)+(\hbar n) L^{-1}+\sum_{k>1} v^{(k)} L^{-k}
$$

The compatibility equations

$$
\hbar \frac{\delta}{\delta A(z)} H\left(z^{\prime}\right)-\hbar \frac{\delta}{\delta A\left(z^{\prime}\right)} H(z)=\left[H(z), H\left(z^{\prime}\right)\right]
$$

determine evolution of $L$ (i.e., the coefficients $u_{n}^{(k)}$ in (11)).

Eqs. (25,27) describe an evolution with respect to deformation parameters, while Eq. (12) describe the growth (increasing $N$ while keeping all harmonic moments fixed).

Equations $(8,25,26,27)$ constitute the Lax-Sato form of the Toda lattice integrable hierarchy [25]. Biorthogonal polynomials form a very particular solution of the hierarchy. If $A(z)$ is a rational function, the matrices $H(z)$ acquire a special structure, which is determined by poles and residues of $A(z)$. This case corresponds to finite-dimensional reductions of the Toda lattice hierarchy.

\subsection{Evolution of the semiclassical curve. Whitham hierarchy}

The variation of the Schwarz function, or the generating differential (22), under a change of the deformation parameters is a subject of deformation equations. The deformation equations are already built in the decomposition of the Schwarz function (23).

In order to write them, we need holomorphic and meromorphic differentials canonically normalized with respect to the a-cycles; they are defined in the following: 
(i) Holomorphic differentials (Abelian differentials of the first kind) $d W_{\alpha}(z)$, $\oint_{\mathbf{a}_{\alpha}} d W_{\beta}=\delta_{\alpha \beta}$

(ii) Meromorphic differentials (Abelian differentials of the third kind) $d W^{(\zeta, \bar{\zeta})}(z)$ having poles at $z=\zeta$ and its mirror on the back side with the residues \pm 1 , normalized so that $\oint_{\mathbf{a}} d W^{(\zeta, \bar{\zeta})}(z) d z=0$. The meromorphic differential $d W^{(\infty, \bar{\infty})}(z)$ plays a special role.

The variation of $d \Omega=S d z$ with respect to $t=\hbar N$ gives the meromorphic differential $d W^{(\infty, \bar{\infty})}(z)$ :

$$
\partial_{t} S(z) d z=-d W^{(\infty, \bar{\infty})}(z)
$$

This follows from the fact that the singular part of the Schwarz function $(A(z)$ in $(23))$ does not depend on $t$, and that $S_{-}(z) \rightarrow t / z$ at infinity.

Variations with respect to the filling factors $t^{(\alpha)}=\hbar \nu_{\alpha}$ at a fixed $t$ and a fixed potential affects only the $S(z)-t / z$ part of the Schwarz function. It gives some $g$ - holomorphic differentials. We call them $d W_{\alpha}(z)$ :

$$
\partial_{t^{(\alpha)}} S(z) d z=-d W_{\alpha}(z)
$$

These differentials are canonically normalized: $\oint_{\mathbf{a}_{\alpha}} d W_{\beta}=\delta_{\alpha \beta}$, so they are unique.

Finally, a variation of $d \Omega=S d z$ with respect to $A(\zeta)$, at fixed filling factors, produces a meromorphic differential which has simple poles at $z=\zeta, \infty$ and their mirrors on the back side. The poles come from the variation of the first term of (23). Their residues are \pm 1 and the sum of the residues is zero on each side of the double. Since all filling factors are fixed, this differential has zero a-periods and we get

$$
\frac{\delta}{\delta A(\zeta)} S(z) d z=-d W^{(\zeta, \bar{\zeta})}(z)+d W^{(\infty, \bar{\infty})}(z), \quad \text { on the front side. }
$$

This equation simply reflects the fact that all singularities of the Schwarz function are the singularities of $A(z)$.

Compatibility equations constitute exchange relations among the differentials. They have the form of the Whitham hierarchy [26]:

$$
\left\{\begin{aligned}
\nabla(\zeta) W^{(\xi, \bar{\xi})}(z) & =\nabla(\xi) W^{(\zeta, \bar{\zeta})}(z) \\
\nabla(\zeta) W^{(\infty, \bar{\infty})}(z) & =\partial_{t} W^{(\zeta, \bar{\zeta})}(z) \\
\nabla(\zeta) W_{\alpha}(z) & =\partial_{t^{(\alpha)}} W^{(\zeta, \bar{\zeta})}(z)
\end{aligned}\right.
$$

where $\nabla(z)=\partial_{t}+\frac{\delta}{\delta A(z)}+\frac{\delta}{\delta \overline{A(z)}}$. 
Now we can check that the Whitham equations (30) are the semiclassical limit of the quantum deformation equations (27). Substituting the semiclassical form of the wave function (21) into (25), we see that the classical limit of the flows are primitive functions of the meromorphic differentials $d W^{(\xi, \bar{\xi})}(z)$ :

$$
H_{n m}(\xi) \rightarrow W^{(\xi, \bar{\xi})}(z)
$$

Similarly, the classical limit of the shift operator $\hat{w}$ defined in Sec. 2 is equal to $W^{(\infty, \bar{\infty})}(z)$, and the second equation of (30) is a classical limit of the compatibility conditions.

The deformation equations become more illustrative for algebraic domains [3] and especially significant for simply-connected algebraic domains [1]. In these cases all the differentials are expressed through the Green function for the exterior Dirichlet boundary problem. Differentials of the third kind are expressed through the Green function on the physical sheet,

$$
d W^{(\zeta, \bar{\zeta})}(z)=2 \partial_{z} G(\zeta, z) d z
$$

while the holomorphic differentials are related to harmonic measures of the droplets:

$$
d W_{\alpha}(z)=\partial_{z} \omega_{\alpha}(z) d z
$$

We recall that the Green function $G(\zeta, z)$ is a symmetric and harmonic function everywhere in the exterior of $D$ except at $z=\zeta$, where it has a logarithmic singularity, $G(\zeta, z) \sim \log |\zeta-z|$. The Green function vanishes if any of the arguments belongs to the boundary. The harmonic measure of a droplet $D_{\alpha}$ is a harmonic function in the exterior of $D$ such that it is equal to 1 on the boundary of $D_{\alpha}$ and vanishes at boundaries of all other droplets.

If there is only one physical droplet, and no virtual droplets, the Green function is given by a conformal map $w(z)$ of the exterior of the droplet to the exterior of the unit disc: $G(\zeta, z)=\log \left|\frac{w(\zeta)-w(z)}{1-w(\zeta) \overline{w(z)}}\right|$. In this case the deformation equations read

$$
\nabla(\zeta) S(z)=-2 \partial_{z} G(\zeta, z), \quad \partial_{t} S(z)=\partial_{z} \log w(z) .
$$

The second equation in (31) describes the evolution of the conformal map when the area of the domain increases, while all the harmonic moments stay constant. This equation, in different equivalent forms, has been known in the theory of Laplacian growth for a long time [27]. The first equation (31) appeared in $[1,23,28]$; for its generalization for multiply-connected algebraic domains, see [29]. A generalization of deformation and growth equations for the Laplacian growth of multiply-connected domains was reported in Ref.[3]. It appeares that they constitute the same Whitham hierarchy as the semiclassical limit of the evolution of the normal matrix ensemble (30). 


\section{Appendix. Laplacian Growth}

Laplacian growth referres to growth of a planar domain, whose boundary propagates with a velocity proportional to the gradient of a harmonic field. The Hele-Shaw problem is a typical example. It describes the dynamics of a $2 \mathrm{D}$ system of two immiscible fluids (such as oil and water). The fluids are confined between two horizontal glass plates, separated by a small distance, so that the problem is essentially two-dimensional.

The motion of the interface follows from the Navier-Stokes equation being specified for a cell. It reads: velocity field of oil is proportional to gradient of pressure. The liquids are assumed to be incompressible, so that the pressure is a harmonic function.

In Refrs. [1,2,3], we identified the Laplacian growth with the Whitham hierarchy. It appeares to be identical to equations (30) describing the growth and deformation of the support of eigenvalues of the normal matrix ensemble.

\section{Acknowledgments}

We are indebted to A. Kapaev, V. Kazakov, I. Krichever, I. Kostov, A. Marshakov and M. Mineev-Weinstein for useful discussions, interest in the subject and help. P.W. and R.T. were supported by the NSF MRSEC Program under DMR-0213745, NSF DMR-0220198 and by the Humboldt foundation. A.Z. and P.W. acknowledge support by the LDRD project 20020006ER "Unstable Fluid/Fluid Interfaces" at Los Alamos National Laboratory and M. Mineev-Weinstein for the hospitality in Los Alamos. A.Z. was also supported in part by RFBR grant 03-02-17373,w and by the grant for support of scientific schools NSh-1999.2003.2. P.W. is grateful to K.B. Efetov for the hospitality in RuhrUniversitaet Bochum and to A.Cappelli for the hospitality in the University of Florence.

\section{References}

[1] Mineev-Weinstein, M., Wiegmann, P.B., \& Zabrodin, A., 2000, Phys. Rev. Lett. $\mathbf{8 4}, 5106$.

[2] Agam, O., Bettelheim, E., Wiegmann, P.B., \& Zabrodin, A., Viscous Fingering and a Shape of an Electronic Droplet in a Quantum Hall Regime, 2002, Phys. Rev. Lett. 88, 236802. 
[3] Krichever, I., Mineev-Weinstein, M., Wiegmann, P.B., \& Zabrodin, A., Laplacian growth and Whitham Equations of Soliton Theory, 2003, nlin.SI/0311005

[4] Teodorescu, R., Bettelheim, E., Agam, O., Zabrodin, A., \& Wiegmann, P., Normal random matrix ensemble as a growth problem, 2004, submitted to Nuclear Physics B, hep-th/0401165

[5] Dijgraaf, R., \& Vafa, C., $\mathcal{N}=1$ Supersymmetry, Deconstruction and Bosonic Gauge Theory, 2003, hep-th/0302011, A Perturbative Window into Non-Perturbative Physics, 2002, hep-th/0208048, Matrix Models, Topological Strings and Supersymmetric Gauge Theories, 2002, hep-th/0206255, On Geometry and Matrix Models, 2002, hep-th/0207106.

[6] Bertola, M., Eynard, B. \& Harnad, J., Partition functions for Matrix Models and Isomonodromic Tau Functions, 2002, nlin/0204054.

[7] Kapaev, A., Riemann-Hilbert problem for bi-orthogonal polynomials, 2003, J. Phys. A 36, 4629-4640.

[8] Di Francesco, P., Ginsparg, P., \& Zinn-Justin, J., 2-D Gravity and Random Matrices, 1995, Phys. Rept. 254, 1-133.

[9] Fokas, A.S., Its, A.R., \& Kitaev, A., The isomonodromy approach to the matrix models in 2-D gravity, 1992, Comm. Math. Phys. 147, 395-430.

[10] Kazakov, V.A., \& Marshakov, A., Complex Curve of the Two Matrix Model and its Tau-function, 2003, J. Phys. A 36, 3107-3136.

[11] Chekhov, L., \& Mironov, A., Matrix Models vs. Seiberg-Witten/Whitham theories, 2002, hep-th/0209085.

[12] Chau, Ling-Lie, \& Zaboronsky, O., On the structure of Normal Matrix Model, 1998, Commun. Math. Phys. 196, 203-247, hep-th/9711091

[13] Mehta, M.L., Random Matrices, 1991, Academic Press, New York.

[14] Wiegmann, P.B., \& Zabrodin, A., Large Scale Correlations in Normal and General Non-hermitian Matrix Ensembles, 2003, J. Phys. A, 36, 3411-3424.

[15] Aratyn, H., Integrable Lax Hierarchies, their Symmetry Reductions and MultiMatrix Models, 1995, hep-th/9503211.

[16] Bertola, M., Eynard B., \& Harnad, J., Duality of spectral curves arising in two-matrix models, 2001, nlin/0112006.

[17] Bertola, M., Eynard, B., \& Harnad, J., Differential systems for biorthogonal polynomials appearing in 2-matrix models and the associated Riemann-Hilbert problem, 2002, nlin/0208002.

[18] Wasow, W., Asymptotic Expansions for Ordinary Differential Equations, 1965, John Wiley \& Sons, New York. 
[19] Dubrovin, B.A., Krichever, I.M., \& Novikov, S.P., Topological and algebraic geometry methods in contemporary mathematical physics II, 1982, Soviet Scient. Reviews, Math. Phys. Reviews 3 1-150;

Krichever, I.M., Integration of nonlinear equations by the method of algebraic geometry, 1977, Funct. Anal. Appl., 11, 12-26.

[20] Ahlfors, L.V., Complex Analysis, an Introduction to the Theory of Analytic Functions of One Complex Variable, 1953, McGraw-Hill, New York.

[21] Cohn, H., Conformal Mapping on Riemann Surfaces, 1967, Dover, New York.

[22] Schiffer, M., \& Spencer, D.C., Functionals of finite Riemann surfaces, 1954, Princeton University Press.

[23] Kostov, I.K., Krichever, I., Mineev-Weinstein, M., Wiegmann, P.B., \& Zabrodin, A., $\tau$-Function for Analytic Curves, in: Random matrices and their applications, 2000, MSRI publications, vol. 40, 285, Cambridge University Press.

[24] Marshakov, A., Mironov, A., \& Morozov, A., Generalized matrix models as conformal field theories: Discrete case, 1991, Phys. Lett. B, 265, 99;

Kharchev, S., Marshakov, A., Mironov, A., Morozov, A., \& Pakuliak, S., Conformal matrix models as an alternative to conventional multimatrix models, 1993, Nucl. Phys. B 404, 717, arXiv:hep-th/9208044.

[25] Ueno, K., \& Takasaki, K., 1984, Adv. Stud. Pure Math., 4, 1.

[26] Whitham, J.B., Linear and nonlinear waves, Wiley-Interscience, 1974, New York;

Flaschka, H., Forest, M.G., \& McLaughlin, D.W., Multiphase averaging and the inverse spectral solution of the Korteweg-de Vries equation, 1980, Comm. Pure Appl. Math., 33, 739-84;

Krichever, I.M., Method of averaging for two-dimensional "integrable" equations, 1988, Funct. Anal. Appl., 22, 200-213.

[27] Galin, L.A., 1945, Dokl. Akad. Nauk SSSR, 47, 250-253;

Polubarinova-Kochina, P.Ya., 1945, Dokl. Akad. Nauk SSSR, 47, 254-257;

Kufarev, P.P., 1947, Dokl. Akad. Nauk SSSR 57, 335-348.

[28] Wiegmann, P.B., \& Zabrodin, A., Conformal maps and dispersionless integrable hierarchies, 2000, Commun.Math.Phys. 213, 523-538;

Marshakov, A., Wiegmann, P.B., \& Zabrodin, A., Integrable Structure of the Dirichlet Boundary Problem in Two Dimensions, 2002, Commun. Math. Phys. 227, 131-153.

[29] Krichever, I., Marshakov, A., \& Zabrodin, A., Integrable Structure of the Dirichlet Boundary Problem in Multiply-Connected Domains, 2003, hep-th/0309010. 